\title{
A GROUP OF EXPONENT 4 WITH DERIVED LENGTH AT LEAST 4
}

\author{
S. BACHMUTH, H. Y. MOCHIZUKI ${ }^{1}$ AND K. WESTON
}

Abstract. There exists a group $G$ of exponent 4 such that the third term of its derived series, $\delta_{3}(G)=G^{m}$, is nontrivial. Furthermore, the third term of the derived series of $G$ is not contained in the ninth term of its lower central series.

1. Introduction. Let $G=F_{\infty}\left(B_{4}\right)$ be the free group of exponent 4 and of countable rank. It has long been conjectured that $G$ is solvable. Recently, N. D. Gupta and K. Weston [4] showed that the solvability of $G$ is equivalent to the solvability of a group $H$, generated by a countable number of generators, each of order 2, and satisfying certain other properties. This group $H$ is a homomorphic image of $G$. We have established that the third term of the derived series of $H$ is nontrivial. As a by-product of our computations, we were also able to answer the question considered by A. L. Tritter in [6] by establishing that the third term of the derived series of $G$ is not contained in the ninth term of the lower central series of $G$.

These facts are in the direction of the conjecture of the authors that $G$ is in fact nonsolvable.

2. Reduction of the problem. Let $(g, h)=g^{-1} h^{-1} g h$ and $(g, h, k)=$ $((g, h), k)$ for elements in a group. $H$ will denote the group generated by $g_{1}, g_{2}, \cdots$ and satisfying the following conditions:

For each $i=1,2, \cdots$

(i) $g_{i}^{2}=1$,

(ii) the normal closure of $g_{i}$ in $H$ is abelian, and

(iii) $\left(g_{i}, h, h, h\right)=1$ for all $h$ in $H$.

In any group $\delta_{k}$ will mean the $k$ th term of the derived series and $\gamma_{k}$ the $k$ th term of the lower central series. Thus $\delta_{0}(H)=H, \delta_{1}(H)=H^{\prime}, \cdots$, and $\gamma_{1}(H)=H, \gamma_{2}(H)=H^{\prime}, \cdots$.

THEOREM. $\quad \delta_{3}(H) \neq 1$, i.e., the third derived group of $H$ is nontrivial.

Received by the editors September 11, 1972.

AMS (MOS) subject classifications (1970). Primary 20E10, 20E35, $20 \mathrm{~F} 45$.

Key words and phrases. Group of exponent 4, derived length.

1 Research supported by NSF Grant GP-29080X.

(c) American Mathematical Society 1973 
With little additional effort, our methods yielded the corollaries:

CoRollary $1 . \quad \delta_{3}(H)$ 查 $\gamma_{9}(H)$.

CoRollary 2. $\delta_{3}(G)$ is not in the center of $G=F_{\infty}\left(B_{4}\right)$.

The proof of the theorem depends on computer computations. The purpose of this section is to simplify the problem in order to make the computations feasible as regards computer space and time. Similar simplifications facilitated the proof of the existence of a nonsolvable group of exponent 5 (see [2]). In the end, the computer time required to prove the results presented here was less than one and one-half hours (on an IBM 360). We refer to the appendix for a discussion of these matters.

The construction below comes from a useful method of constructing multiplicative groups with exponent in an associative ring (see R. Bruck [3, pp. S4.5 and S4.6]). The particular ring employed below essentially appears in Gupta and Weston [4, §4]. In the most general context, these rings may be considered as homomorphic images of group rings modulo "cyclotomic ideals". These ideals were defined and studied in certain abelian group rings in [1] where some striking results were obtained. For the nonabelian case our knowledge of these ideals is virtually nil. Viewed in this context, the results presented here as well as in [2] may be considered a start in this general direction. For motivation of these problems as well as some details which we omit below, we recommend Bruck's notes [3] and also $\S 4$ of the exponent 5 paper [2].

Let $R$ be the free associative ring (with 1 ) of characteristic 2 which is generated by noncommuting indeterminates $x_{1}, x_{2}, \cdots$. Let $\mathscr{J}$ be the ideal of $R$ generated by all the monomials $x_{i_{1}} x_{i_{2}} \cdots x_{i_{m}}$ in which for some $j \neq k, i_{j}=i_{k}$, and let $\mathscr{T}$ be the ideal of $R$ generated by all the polynomials $T_{3}\left(x_{i_{1}}, x_{i_{2}}, \cdots, x_{i_{m}}\right), m \geqq 3$, where $T_{3}\left(x_{1}, \cdots, x_{n}\right)$ is the homogeneous component of degree one in each of the $x_{i}$ of

$$
\left[\left(1+x_{1}\right)\left(1+x_{2}\right) \cdots\left(1+x_{n}\right)-1\right]^{3} \text {. }
$$

Let $r_{i}=x_{i}+(\mathscr{J}+\mathscr{T})$ in $R /(\mathscr{J}+\mathscr{T})$. Then $1+r_{1}, 1+r_{2}, \cdots$ generate a (multiplicative) group $H^{*}$ which is a homomorphic image of $H$ under the map $g_{i} \rightarrow 1+r_{i}, i=1,2, \cdots$. Thus, for $i=1,2, \cdots$,

(i) $\left(1+r_{i}\right)^{2}=1$,

(ii) the normal closure of each $\left(1+r_{i}\right)$ in $H^{*}$ is abelian, and

(iii) $\left(1+r_{i}, u, u, u\right)=1$ for all $u$ in $H^{*}$.

As in [4], we often shall use $1,2, \cdots$ in place of $r_{1}, r_{2}, \cdots$ respectively. We let $\Delta(1,2)=12+21$ and inductively,

$$
\begin{aligned}
\Delta\left(1,2, \cdots, 2^{k+1}\right)= & \Delta\left(1,2, \cdots, 2^{k}\right) \Delta\left(2^{k}+1, \cdots, 2^{k+1}\right) \\
& +\Delta\left(2^{k}+1, \cdots, 2^{k+1}\right) \Delta\left(1,2, \cdots, 2^{k}\right),
\end{aligned}
$$


for the derived ring commutators. We put

and

$$
w\left(1+r_{1}, 1+r_{2}\right)=\left(1+r_{1}, 1+r_{2}\right)
$$

$$
\begin{aligned}
w\left(1+r_{1},\right. & \left.\cdots, 1+r_{2^{k+1}}\right) \\
& =\left(w\left(1+r_{1}, \cdots, 1+r_{2^{k}}\right), w\left(1+r_{2^{k}+1}, \cdots, 1+r_{2^{k+1}}\right)\right)
\end{aligned}
$$

for the derived group commutators. It then follows that

$$
w\left(1+r_{1}, \cdots, 1+r_{2^{k}}\right)=1+\Delta\left(1,2, \cdots, 2^{k}\right) .
$$

We show that $\Delta(1,2, \cdots, 8) \neq 0$ in $R /(\mathscr{J}+\mathscr{T})$, i.e., $H^{*}$ has derived length $\geqq 4$.

With some ambiguity we will also use $1,2, \cdots$ in place of $x_{1}, x_{2}, \cdots$. Thus, we want to prove that $\Delta(1,2, \cdots, 8)$ is not in $(\mathscr{J}+\mathscr{T})$. Since $R$ is the direct sum of its homogeneous parts and $\Delta(1,2, \cdots, 8)$ is homogeneous of degree 1 in each of $1,2, \cdots, 8, \Delta(1,2, \cdots, 8)$ is not in $(\mathscr{J}+\mathscr{T})$ if and only if $\Delta(1,2, \cdots, 8)$ is not in $\mathscr{T}$ (cf., R. Bruck [3, p. S4.5]).

$\mathscr{T}$ has a set of generators as a vector space over $Z_{2}$, the field of two elements, consisting of $M_{1} T_{3}\left(i_{1}, \cdots, i_{m}\right) M_{2}, M_{1}$ and $M_{2}$ monomials. Since

$$
\begin{aligned}
(m+1) T_{3}(1,2, \cdots, m)= & T_{3}(1,2, \cdots, m)(m+1) \\
& +\sum_{i=1}^{m} T_{3}(1, \cdots, i-1, m+1, i, \cdots, m) \\
& +T_{3}(1, \cdots, i, m+1, i+1, \cdots, m)
\end{aligned}
$$

(see R. Bruck [3]), $\mathscr{T}$ is generated over $Z_{2}$ by $T_{3}\left(i_{1}, i_{2}, \cdots, i_{m}\right) M, M$ a monomial.

In the following section we will use an equivalent form of the $T_{3}\left(i_{1}, i_{2}, \cdots, i_{m}\right)$ due to Tah-Zen Yuan [7]. This new form also has the property that the right (or left) ideal generated by the equivalent form of the $T_{3}()$ is $\mathscr{T}$.

Let $\varphi$ be the ring homomorphism of $R$ into $R$ defined by

$$
\left(x_{2 i-1}\right) \varphi=x_{i} \text { and }\left(x_{2 i}\right) \varphi=x_{i} \text { for } i=1,2,3, \cdots .
$$

Clearly, $\varphi$ maps $\mathscr{T}$ into $\mathscr{T}$. We show in the next section that

$$
\varphi(\Delta(1,5,2,7,3,6,4,8))=\Delta(1,3,1,4,2,3,2,4)
$$

is not in $\mathscr{T}$.

3. Computer methods. (2.1) is in $\mathscr{T}$ if and only if (2.1) lies in $S \cap \mathscr{T}$ where $S$ is the homogeneous component of $R$ of degree 2 in each of $1,2,3,4$ and of total degree 8 . 
The monomials in $S$ were generated and ordered lexicographically by the computer. $11223344<11223434<11223443<\cdots$. There are 2520 such monomials, and these were stored in an array at the start of the program. We now explain how the computer selected a basis for $S \cap \mathscr{T}$ and then verified that $\Delta(1,3,1,4,2,3,2,4)$ is not a linear combination of this basis.

For convenience, we used the reduction of the $T_{3}(1,2, \cdots, k)$ due to Tah-Zen Yuan [7] which we describe now. Let $P=12 \cdots(m-2)$ and $\bar{P}=(m-2) \cdots 21$ its reversal. For $m \geqq 3$,

$$
\begin{aligned}
T_{3}(1,2, \cdots, m)= & P(m-1) m+(m-1) m P+\bar{P} m(m-1) \\
& +(m-1) \bar{P} m+m(m-1) \bar{P}+m \bar{P}(m-1) \\
& +\sum_{\sigma} \sigma(m-1) m \sigma^{\prime}
\end{aligned}
$$

where the last expression is summed over all proper increasing subsequences $\sigma$ of $1,2, \cdots,(m-2)$ and $\sigma^{\prime}$ is the subsequence complementary - to $\sigma$, i.e., $\sigma=i_{1} i_{2} \cdots i_{k}$ where $1 \leqq i_{1}<i_{2}<\cdots<i_{k} \leqq(m-2)$ and $1 \leqq k<$ $(m-2), \sigma^{\prime}=j_{1} j_{2} \cdots j_{l}, 1 \leqq j_{1}<\cdots<j_{l} \leqq(m-2)$ and the variables in $\sigma$ and $\sigma^{\prime}$ together are precisely all the variables 1 through $(m-2)$ each appearing in exactly one of $\sigma$ or $\sigma^{\prime}$. For example,

$$
\begin{aligned}
T_{3}(1,2,3)= & 123+231+132+213+321+312, \\
T_{3}(1,2,3,4)= & 1234+3412+2143+3214+4321+4213 \\
& +1342+2341, \\
T_{3}(1,2,3,4,5)= & 12345+45123+32154+43215+54321+53214 \\
& +14523+24513+34512+12453+13452+23451, \\
& \text { etc. }
\end{aligned}
$$

The following six expressions were stored in the computer.

$$
\begin{aligned}
& T_{3}(1,2,3) 45678, \\
& T_{3}(1,2,3,4) 5678, \\
& T_{3}(1,2,3,4,5) 678, \\
& T_{3}(1,2,3,4,5,6) 78, \\
& T_{3}(1,2,3,4,5,6,7) 8, \\
& T_{3}(1,2,3,4,5,6,7,8) .
\end{aligned}
$$

Also, a $2520 \times 2520$ section of core was set aside to form a matrix $M$, the columns of $M$ being indexed by the ordered monomials of $S$.

We used the list of 2520 stored monomials of $S$ to substitute for the letters $1,2, \cdots, 8$ in (3.1) to obtain a complete list of generators for 
$S \cap \mathscr{T}$. For example, if $k_{1} k_{2} \cdots k_{8}$ is the $k$ th monomial in the list, then $k_{1}, k_{2}, \cdots, k_{8}$ were substituted for the $1,2, \cdots, 8$ respectively in each of the six expressions of (3.1). Each of these six generators (in turn) was then checked against the (upper triangular) matrix $M$ to see whether or not it formed a new basis element for $S \cap \mathscr{T}$. If so, it was placed into $M$; otherwise it was discarded. This procedure, after processing all $r=2520 \times 6$ generators, produced an upper triangular matrix $M$, whose row vectors formed a basis for $S \cap \mathscr{T}$. Specifically, the procedure was as follows:

Suppose now we are at the $r$ th step. The matrix $M$ has certain nonzero entries obtained by the preceding $(r-1)$ steps. The $m$ th row of $M$ is nonzero if and only if the $(m, m)$ entry is 1 . If the $m$ th row is nonzero and the nonzero entries are in the $m, n, \cdots, p$ columns, $m<n<\cdots<p$, then $N(m)+N(n)+\cdots+N(p)$ is a basis element of $S \cap \mathscr{T}$, where $N(j)$ denotes the $j$ th monomial in our list of monomials of $S$.

We now consider the $r$ th generator, say

$$
N(i)+N(j)+\cdots+N(s), \quad i<j<\cdots<s .
$$

If the $(i, i)$ entry of $M$ is zero, insert into the $i$ th row of $M$ the entries which have 1 in the $i, j, \cdots, s$ columns and zero elsewhere. This means of course that (3.2) is a new basis element and has been so recorded in $M$. Suppose on the other hand, the $(i, i)$ entry of $M$ is 1 , say the $i$ th row represents the basis element

$$
N(i)+N(l)+\cdots+N(t), \quad i<l<\cdots<t .
$$

Then the elements (3.2) and (3.3) are added together to eliminate the $N(i)$ term and to obtain either zero or a new nonzero generator. We continue this elimination process until we obtain either a new basis element which is then inserted into $M$, or else we obtain zero which means that (3.2) is a linear combination of basis elements already found and recorded in $M$.

We then continue to the $(r+1)$ th generator. This process continues until all $2520 \times 6$ generators have been processed, and we thus end up with a triangular matrix $M$ whose rows represent a basis for $S \cap \mathscr{T}$.

With the completed matrix $M$, it is now a matter of seconds for the computer to check whether a given element of $S$ is in $\mathscr{T}$ or not. We showed that the element

$$
\varphi(\Delta(1,5,2,7,3,6,4,8))=\Delta(1,3,1,4,2,3,2,4)
$$

is not a linear combination of the basis elements of $S \cap \mathscr{T}$. This means that $\Delta(1,5,2,7,3,6,4,8)$ cannot be in $\mathscr{T}$ and hence $H^{*}$ has derived length $\geqq 4$.

Corollary 1. $\delta_{3}(H) \gamma_{9}(H)$. 
Proof. Let $H(8)$ be the subgroup of $H$ generated by $g_{1}, g_{2}, \cdots, g_{8}$. $\gamma_{9}(H(8))=1$ but $\delta_{3}(H(8)) \neq 1$. Under the homomorphism $g_{i} \rightarrow g_{i}, 1 \leqq i \leqq 8$, $g_{j} \rightarrow 1, j=9,10, \cdots, \gamma_{9}(H)$ is mapped into 1 whereas $\delta_{3}(H)$ is not mapped to 1 . Thus, the corollary follows.

Corollary 2. $\delta_{3}(G)$ is not in the center of $G$.

Proof. In the ring of $2 \times 2$ matrices over $R /(\mathscr{J}+\mathscr{T})$, consider the group $K$ generated by

$$
X=\left(\begin{array}{ll}
1 & 0 \\
1 & 1
\end{array}\right), \quad Y_{i}=\left(\begin{array}{cc}
1+r_{i} & 0 \\
r_{i} & 1
\end{array}\right), \quad i=1,2, \cdots
$$

It is easily verified that $K$ is a group of exponent 4 generated by elements of order 2 .

$$
\left(X, w\left(Y_{1}, \cdots, Y_{8}\right)\right)=\left(\begin{array}{cc}
1 & 0 \\
\Delta\left(r_{1}, \cdots, r_{8}\right) & 1
\end{array}\right) \neq\left(\begin{array}{ll}
1 & 0 \\
0 & 1
\end{array}\right) .
$$

Thus, $\delta_{3}(K)$ is not contained in the center of $K$.

REMARK. A group construction similar to the group $K$ of Corollary 2 was used in an earlier work by Gupta, Mochizuki and Weston [5].

Appendix. The computer used was an IBM-360. Steve Smith wrote the final program in machine language so effectively that the entire run was under one and one-half hours. One reason for so short an execution time was that auxiliary storage space was not required. The matrix $M$ required half of $(2520)^{2}$ memory locations because $M$ was always triangular. In addition a packing routine was employed which enabled four entries of $M$ to be stored in a single memory location. This further reduced the storage requirements for $M$ by a factor of four, and as a result the memory location for the complete program was accessible from the (relatively) high speed main core.

The final matrix $M$ which contains the basis for $S \cap \mathscr{T}$ has been stored on tape. The computer program and the relational matrix $M$ is on file at the Computer Center at UCSB.

Acknowledgments. We wish to thank Mr. Steve Smith of the Computer Center of the University of California, Santa Barbara (UCSB), for his exceptional programming work, the Research Committee of UCSB for computer funds, and the Computer Center, UCSB, for use of their facilities.

\section{REFERENCES}

1. S. Bachmuth, H. A. Heilbronn and H. Y. Mochizuki, Burnside metabelian groups, Proc. Roy. Soc. A. 307 (1968), 235-250. MR 38 \#4561.

2. S. Bachmuth, H. Y. Mochizuki and D. W. Walkup, Construction of a nonsolvable group of exponent 5, Word Problems (edited by W. W. Boone, R. C. Lyndon, F. B. Cannonito), North-Holland, Amsterdam. (to appear). 
3. R. H. Bruck, Engel conditions in groups and related questions, Lecture Notes, Australian National University, Canberra, Jan. 1963.

4. N. D. Gupta and K. Weston, On groups of exponent four, J. Algebra 17 (1971), 59-66. MR 42 \#3176.

5. N. D. Gupta, H. Y. Mochizuki and K. Weston, On groups of exponent four with generators of order two, J. Austral Math. Soc. (to appear).

6. A. L. Tritter, A module-theoretic computation related to the Burnside problem, Computational Problems in Abstract Algebra, Proc. Conference (Oxford, 1967), Pergamon Press, Oxford, 1970, pp. 189-198. MR 41 \#6981.

7. Tah-Zen Yuan, Thesis, University of Notre Dame, South Bend, Ind., 1969.

Department of Mathematics, University of California, Santa Barbara, California 93106

Current address: Department of Mathematics, University of Wisconsin, Kenosha, Wisconsin 53140 